\title{
PAPER
}

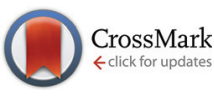

Cite this: Food Funct., 2014, 5, 2922

\section{Walnut polyphenol metabolites, urolithins A and B, inhibit the expression of the prostate-specific antigen and the androgen receptor in prostate cancer cells}

\author{
Claudia Sánchez-González, ${ }^{a}$ Carlos J. Ciudad, ${ }^{\mathrm{b}}$ Véronique Noéb and \\ Maria Izquierdo-Pulido ${ }^{a, c}$
}

\begin{abstract}
Walnuts have been gathering attention for their health-promoting properties. They are rich in polyphenols, mainly ellagitannins (ETs) that after consumption are hydrolyzed to release ellagic acid (EA). EA is further metabolized by microbiota to form urolithins, such as A and B, which are absorbed. ETs, EA and urolithins have shown to slow the proliferation and growth of different types of cancer cells but the mechanisms remain unclear. We investigate the role of urolithins in the regulatory mechanisms in prostate cancer, specifically those related to the androgen receptor (AR), which have been linked to the development of this type of cancer. In our study, urolithins down-regulated the mRNA and protein levels of both prostate specific antigen (PSA) and AR in LNCaP cells. The luciferase assay performed with a construct containing three androgen response elements (AREs) showed that urolithins inhibit AR-mediated PSA expression at the transcriptional level. Electrophoretic mobility shift assays revealed that urolithins decreased AR binding to its consensus response element. Additionally, urolithins induced apoptosis in LNCaP cells, and this effect correlated with a decrease in Bcl-2 protein levels. In summary, urolithins attenuate the function of the AR by repressing its expression, causing a down-regulation of PSA levels and inducing apoptosis. Our results suggest that a diet rich in ET-containing foods, such as walnuts, could contribute to the prevention of prostate cancer.
\end{abstract}

Received 19th June 2014 Accepted 18th August 2014

DOI: $10.1039 / c 4 f 000542 b$

www.rsc.org/foodfunction such as reducing the pro-oxidative effect of carcinogenic agents, ${ }^{8,9}$ modulation of cancer cell signaling, ${ }^{10,11}$ cell cycle progression, ${ }^{12,13}$ promotion of apoptosis, ${ }^{14,15}$ and modulation of enzymatic activities. ${ }^{16}$ Regarding prostate cancer progression, a recent clinical trial assessed the effect of a polyphenol-blend dietary supplement over prostate-specific antigen (PSA) levels in men with localized prostate carcinoma; this study found a significant favorable effect on the percentage rise in PSA levels, an important indicator of prostate cancer progression. ${ }^{17}$ Polyphenols have also been shown to act on multiple targets in pathways not only related to cancer progression, cellular proliferation and death, ${ }^{18}$ but also in inflammation, ${ }^{19}$ angiogenesis, ${ }^{20}$ and drug and radiation resistance. ${ }^{21}$

Walnuts (Juglans regia L.) have been gathering increasing attention for their health-promoting properties, which have been reported to improve lifestyle-related diseases such as arteriosclerosis, hypercholesterolemia, hypertriglyceridemia, cardiovascular disease, diabetes, and cancer. ${ }^{22-24}$ Walnuts are rich in bioactive polyphenols (total contents ranging from $1575 \mathrm{mg}$ to $2500 \mathrm{mg}$ per $100 \mathrm{~g}$ ) and they represent, on a serving size basis, the seventh largest source of total polyphenols among common foods and beverages. ${ }^{25}$ The most

\footnotetext{
${ }^{a}$ Nutrition and Food Science Department, School of Pharmacy, University of Barcelona, Barcelona, Spain

${ }^{b}$ Biochemistry and Molecular Biology Department, School of Pharmacy, University of Barcelona, Barcelona, Spain

${ }^{C}$ CIBER Fisiopatología de la Obesidad y la Nutrición (CIBEROBN), Spain.

E-mail: maria_izquierdo@ub.edu
} 
abundant polyphenols in walnuts are ellagitannins (ETs), mainly pedunculagin. ${ }^{26}$ ETs are tannins that release ellagic acid (EA) upon hydrolysis, which are further metabolized by gut flora to form urolithins, mainly urolithins $\mathrm{A}$ and $\mathrm{B}^{27}$ These urolithins circulate in blood and can reach many of the target organs where the effects of ellagitannins are noted. ${ }^{27,28}$ Although the occurrence of ETs and EA in the bloodstream is almost negligible, urolithins can reach a concentration at micromolar levels in plasma, ${ }^{29}$ their maximum concentration is reached 24 to 48 hours after consumption of ET-rich foods, although urolithins can be found in plasma and urine up to 72 hours after consumption in both free and conjugated forms $;{ }^{27}$ urolithins and their conjugates have also been found in the human prostate after walnut and pomegranate juice consumption. ${ }^{30}$ Like other polyphenols, ETs, EA and their derived metabolites possess a wide range of biological activities which suggest that they could have beneficial effects on human health. ${ }^{31}$ Moreover, ETs and EA seem to exhibit anticancer properties in vitro and in vivo. Recent research in vitro has shown that walnut extracts have dose-dependent inhibitory effects on colon cancer cell growth ${ }^{32}$ and it has been observed that walnuts delay the growth rate of breast cancer cells ${ }^{33}$ and prostate cancer cells ${ }^{30}$ implanted in mice. ET-rich herbal extracts have been shown to inhibit LNCaP cell proliferation and reduce PSA secretion. ${ }^{34}$ Other authors have also attributed estrogenic and anti-estrogenic activity to urolithins based on their binding affinity to the estrogen receptor in MCF-7 cells, labeling urolithins as potential endocrine-disruptive molecules. ${ }^{29}$

Prostate-specific antigen is a well-known prostate tumor marker, expressed at a high level in the luminal epithelial cells of the prostate and is absent or expressed at very low levels in other tissues. ${ }^{35}$ However recent data suggest that PSA is not only a biomarker, but that it also has a biological role in the development and progression of prostate cancer, since it is involved in tumor growth, invasion and metastasis. ${ }^{36}$ PSA is encoded by the KLK3 gene and its expression is tightly controlled by androgen through the action of the androgen receptor (AR).$^{37}$ Upon binding to androgen, AR translocates into the nucleus and binds to the androgen response elements (AREs) on the PSA promoter, interacting with other transcription factors and activating PSA gene transcription. ${ }^{38}$ The expression of PSA in prostate cancer generally reflects the transcriptional activity of AR, but additional factors regulating the PSA promoter have also been identified. ${ }^{39-41}$

Considering all of the above, we hypothesized that the main walnut polyphenol metabolites, urolithins $\mathrm{A}$ and $\mathrm{B}$, could exert a role over regulatory mechanisms in prostate cancer, specifically those related to the androgen receptor, which have been linked to the development and progression of this type of cancer. To this purpose, and using a prostate cancer cell model (LNCaP cells), we investigated the effects of urolithins A and B on the gene expression of PSA and AR and their protein expression. We also assayed the ability of those compounds to modify the PSA promoter activity and to bind AR. In addition, the effect of both urolithins on apoptosis was also explored.

\section{Experimental}

\subsection{Materials and chemicals}

Urolithin A (UA; 3,8-dihydroxy-6H-dibenzo[b,d]pyran-6-one, $95 \%$ purity) and urolithin B (UB; 3-dihydroxy-6H-dibenzo[b,d]pyran-6-one, $98 \%$ purity) were synthesized by the Department of Organic Chemistry, School of Pharmacy at the University of Barcelona (Barcelona, Spain). Urolithins and dehydrotestosterone (DHT) (Sigma-Aldrich, Madrid, Spain) were suspended in DMSO.

\subsection{Cell culture}

LNCaP (androgen responsive) and PC3 (androgen independent) human prostate adenocarcinoma cell lines were routinely grown in Ham's F-12 medium, supplemented with $7 \%(\mathrm{v} / \mathrm{v})$ fetal bovine serum (FBS, both from GIBCO, Invitrogen, Barcelona, Spain), sodium penicillin $\mathrm{G}$ and streptomycin, and were maintained at $37{ }^{\circ} \mathrm{C}$ under a humidified atmosphere containing 5\% $\mathrm{CO}_{2} .250000-500000$ cells were incubated with $40 \mu \mathrm{M}$ of either urolithin A or urolithin B, or a combination composed of $20 \mu \mathrm{M}$ UA and $20 \mu \mathrm{M}$ UB (named MIX). This concentration was chosen because it can be found in plasma after consumption of ET-rich foods, ${ }^{26-28}$ and it is within the range used to assay the biological activity of urolithins. ${ }^{42,43}$ In addition, this concentration was not cytotoxic (data not shown). Incubations were also performed, depending upon the experiment, with $1 \mathrm{nM}$ of DHT. The final concentration of DMSO in the culture medium was always $\leq 0.5 \%$.

\subsection{RT-real time PCR}

Total RNA was extracted from LNCaP using the Trizol reagent (Life Technologies, Madrid, Spain) in accordance with the manufacturer's instructions. Complementary DNA (cDNA) was synthesized as described by Oleaga et al. (2013). ${ }^{44}$ RNA concentration and purity was checked using a Nanodrop spectrophotometer system (ND-1000 3.3 Nanodrop Technologies, Wilmington, DE, USA). mRNA levels were determined with StepOnePlus $^{\mathrm{TM}}$ real-time PCR systems (Applied Biosystems, Barcelona, Spain) using $3 \mu \mathrm{L}$ of cDNA and Taqman probes (Applied Biosystems, Barcelona, Spain), for KLK3 (Hs02576345) and AR (Hs00171172) genes and APRT (Hs00975725) as an endogenous control. Changes in gene expression were calculated using the quantitative ${ }^{\Delta \Delta} \mathrm{Ct}$ method and normalized against APRT in each sample.

\subsection{Western blot}

LNCaP cells (350000) were plated on $35 \mathrm{~mm}$ dishes and treated the day after with the different compounds. Twentyfour hours after incubation cells were collected and centrifuged for $5 \mathrm{~min}$ at $800 \mathrm{~g}$ at $4{ }^{\circ} \mathrm{C}$. The cell pellets were suspended in $200 \mu \mathrm{L}$ of lysis buffer $\left(0.5 \mathrm{M} \mathrm{NaCl}, 1.5 \mathrm{mM} \mathrm{MgCl}_{2}\right.$, 1 mM EGTA, $10 \%$ glycerol 1\% Triton x_100, 50 mM HEPES, pH 7.9 all from Applichem, Barcelona, Spain), and $10 \mu \mathrm{L}$ protease inhibitor cocktail (from Sigma-Aldrich, Madrid, Spain). The cell lysate was kept on ice for 60 min vortexing every 15 min. Cellular debris was removed by centrifugation at 
$15000 \mathrm{~g}$ at $4{ }^{\circ} \mathrm{C}$ for $10 \mathrm{~min}$. A $5 \mu \mathrm{L}$ aliquot of the extract was used to determine the protein concentration using the Bradford assay (Bio-Rad, Barcelona, Spain).

Whole cell extracts $(100 \mu \mathrm{g})$ were resolved in $12 \%$ SDS-polyacrylamide gels and transferred to PVDF membranes (Immobilon P, Millipore, Madrid, Spain) using a semidry electroblotter. Membranes were probed overnight at $4{ }^{\circ} \mathrm{C}$ with primary antibodies against AR (1:200 dilution; sc-816 from Santa-Cruz Biotechnology Inc., Heidelberg, Germany), PSA (1:300 dilution; A0562 from Dako, Denmark) or Bcl-2 (1: 200 dilution; sc-492 from Santa-Cruz Biotechnology Inc., Heidelberg, Germany). Signals were detected by secondary horseradish peroxidaseconjugated antibody, either anti-rabbit (1:2500; Dako, Denmark) or anti-mouse (1:2500 dilution, sc-2005 Santa Cruz Biotechnology Inc., Heidelberg, Germany) and enhanced chemiluminescence using the ECL ${ }^{\mathrm{TM}}$ Prime Western blotting detection reagent, as recommended by the manufacturer (GE Healthcare, Barcelona, Spain). Chemiluminescence was detected with ImageQuant LAS 4000 Mini technology (GE Healthcare, Barcelona, Spain). Normalization of the blots was performed by incubation with an antibody against tubulin (1: 800 dilution, sc-5286 from Santa-Cruz Biotechnology Inc., Heidelberg, Germany).

\subsection{Transfection and luciferase assay}

PC3 cells (350 000) were plated in $35 \mathrm{~mm}$ dishes the day before transfection. The medium $(2 \mathrm{ml})$ was renewed before transfection, which was performed using FuGENE 6 (Roche, Barcelona, Spain). For each well, the transfection reagent was incubated for 5 minutes in $100 \mu \mathrm{L}$ of antibiotic and serum free medium, followed by the addition of plasmid DNA and incubated for another $20 \mathrm{~min}$ at a ratio of $3: 1$ ( $\mu \mathrm{L}$ of transfection reagent $: \mu \mathrm{g}$ of plasmid DNA). One $\mu \mathrm{g}$ of plasmid DNA, either pGL3 basic vector or PSAp, a $6 \mathrm{~kb}$ PSA promoter construct containing three AREs in front of a luciferase reporter gene were used for transfection.

Incubation with $40 \mu \mathrm{M}$ of UA, UB or MIX and $1 \mathrm{nM}$ of DHT was performed 6 hours after transfection, and the luciferase activity was determined 24 hours after transfection. Cell extracts were prepared by lysing cells with $100 \mu \mathrm{L}$ of reporter lysis buffer (2 mM DTT, 2 mM EDTA, 10\% glycerol, 1\% Triton X_100, 25 mM Tris-phosphate, $\mathrm{pH}$ 7.8). The lysate was centrifuged at $12000 \mathrm{~g}$ for $2 \mathrm{~min}$ at $4{ }^{\circ} \mathrm{C}$ to pellet cell debris and supernatants were transferred to a fresh tube. Fifteen $\mu \mathrm{L}$ of the extract were added to $15 \mu \mathrm{L}$ of the luciferase assay substrate (Promega, Madrid, Spain) at room temperature. Luminescence was measured using the Glomax ${ }^{\mathrm{TM}}$ 20/20 luminometer (Promega, Madrid, Spain) and expressed as relative luminescence units (RLU). Luciferase results were normalized by the total protein concentration in the cell lysates. Protein concentration was determined by the Bradford assay (Bio-Rad, Barcelona, Spain) according to the manufacturer's protocol.

\subsection{Nuclear extracts}

Nuclear extracts were prepared according to the protocol described by Andrews and Faller (1991). ${ }^{45}$ Briefly, 500000 cells were plated and incubated the following day with urolithins A, B or MIX and $1 \mathrm{nM}$ DHT. Cells were collected in cold PBS after being treated for 24 hours. Cells were pelleted and suspended in a cold hypotonic buffer $\left(1.5 \mathrm{mM} \mathrm{MgCl}_{2}, 10 \mathrm{mM} \mathrm{KCl}\right.$ (AppliChem, Barcelona, Spain), 0.5 mM DTT, 0.2 mM PMSF, $10 \mathrm{mM}$ HEPES-KOH, pH 8.0 from Sigma-Aldrich, Madrid, Spain). Cells were then allowed to swell for 10 minutes, vortexed and pelleted by centrifugation. The resulting pellet was then suspended in a cold high-salt buffer (25\% glycerol, $420 \mathrm{mM} \mathrm{NaCl}$, $1.5 \mathrm{mM} \mathrm{MgCl}$, $10 \mathrm{mM} \mathrm{KCl,} 0.5 \mathrm{mM}$ DTT, $0.2 \mathrm{mM}$ PMSF, $20 \mathrm{mM}$ HEPES-KOH, pH 8.0) for 20 minutes. Cellular debris was removed by centrifugation and the supernatant fraction was stored at $-80^{\circ} \mathrm{C}$ until further use.

\subsection{Electrophoretic mobility shift assay}

EMSA assay was performed using LNCaP nuclear extracts prepared as previously described. AR consensus double-stranded oligonucleotide 5'-CTA GAA GTC TGG TAC AGG GTG TTC TTT TTG CA-3' (binding site in bold) was obtained from Santa Cruz Biotechnology, Heidelberg, Germany (sc-2551). One hundred nanograms of the AR consensus sequence was 5 '-end-labeled with T4 polynucleotide kinase (New England Biolabs, Beverly, MA) and $\left[\gamma^{-32} \mathrm{P}\right]$ ATP $\left(3000 \mathrm{Ci} \mathrm{mmol}^{-1}\right.$, Perkin Elmer, Madrid, Spain) as described by Rodríguez et al. (2013). ${ }^{46}$

The radiolabeled probe $(20000 \mathrm{cpm})$ was incubated in a $20 \mu \mathrm{L}$ reaction mixture also containing $1 \mu \mathrm{g}$ of Herring sperm DNA (Invitrogen, Barcelona, Spain) as an unspecific competitor, $2 \mu \mathrm{g}$ of nuclear extract protein, $5 \%$ glycerol, $4 \mathrm{mM} \mathrm{MgCI}_{2}$, $60 \mathrm{mM} \mathrm{KCl}$ and $25 \mathrm{mM}$ Tris-HCl, pH 8.0 (AppliChem, Barcelona, Spain). Samples were resolved by gel electrophoresis $(5 \%$ polyacrylamide, 5\% glycerol, $1 \mathrm{mM}$ EDTA and $45 \mathrm{mM}$ Trisborate, $\mathrm{pH}$ 8.0; AppliChem, Barcelona, Spain). The gel was dried for 90 minutes, exposed to europium plates overnight and analyzed using a Storm 840 Phosphorimager (Molecular Dynamics, GE Healthcare Life Sciences, Barcelona, Spain).

To determine the binding specificity, the radiolabeled ARE probe was competed either with $3 \mathrm{ng}$ (5-fold) of unlabeled ARE consensus or a mutant ARE oligonucleotide. The mutant AR oligonucleotide had two "GT" to "CA" substitutions in the AR binding motif 5'-CTA GAA GTC TGC CAC AGG GTC ATC TTT TTG CA-3' (binding site in bold) (sc-2552, Santa Cruz Biotechnology, Heidelberg, Germany). These experiments were performed using NE from LNCaP cells treated with $1 \mathrm{nM}$ DHT.

\subsection{Apoptosis}

Apoptosis was determined by the rhodamine method. LNCaP cells (250 000) were plated in $35 \mathrm{~mm}$ dishes with $2 \mathrm{ml}$ complete F-12 medium and after $24 \mathrm{~h}$, they were treated with $40 \mu \mathrm{M}$ UA, UB or MIX. Staurosporine $(1 \mu \mathrm{M})$ (Sigma-Aldrich, Madrid, Spain) was used as a positive control. Rhodamine

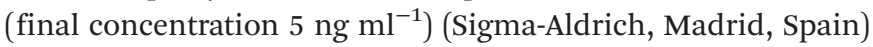
was added for $30 \mathrm{~min}$ and the cells were collected, centrifuged at $800 \mathrm{~g}$ at $4{ }^{\circ} \mathrm{C}$ for $5 \mathrm{~min}$, and washed once with PBS. The pellet was suspended in $500 \mathrm{ml}$ PBS plus propidium iodide (PI) (final concentration $5 \mathrm{mg} \mathrm{m}^{-1}$ ) (Sigma-Aldrich, Madrid, 
Spain). Flow cytometry data were analyzed using the Summit v4.3 software. The percentage of Rho-negative, PI negative cells corresponds to the apoptotic population.

\subsection{Statistical analyses}

All data are reported as mean $\pm \mathrm{SE}$ and are representative of at least three independent experiments. Data were analyzed using one-way ANOVA followed by the Bonferroni post hoc multiple range test using the SPSS software v.21. The difference between groups was considered statistically significant at $p<0.05$.

\section{Results}

\subsection{Urolithins A and B decrease PSA mRNA and protein levels in LNCaP cells}

Taking into account the role of prostate specific antigen in prostate cancer, we analyzed the effect of urolithins on PSA mRNA expression. LNCaP cells were incubated with urolithins during different time periods (12, 24, and $48 \mathrm{~h}$ ). Total RNA was extracted and PSA expression was analyzed by RT-real time PCR (Fig. 1A). On average, urolithins induced the major decrease in PSA mRNA levels after 24 hours; urolithin A provoked an $85 \%$ reduction, a similar effect was observed after incubation with MIX at the same time point, while UB exerted a $50 \%$ inhibition. To examine whether the effects observed at the mRNA level were translated into the protein, we performed Western blot analyses in LNCaP cells after 24 hour incubation with urolithins. As shown in Fig. 1B, cells incubated with UA exhibited a $63 \%$ decrease in PSA protein levels compared to the untreated control, followed by cells treated with MIX or UB.

\subsection{Urolithins A and B decrease AR mRNA and protein expression}

To determine whether urolithins were able to modulate AR mRNA expression, LNCaP cells were incubated for several time periods, between 9 and 24 hours; total RNA was extracted and AR expression was analyzed by RT-real time PCR. A decrease in AR mRNA levels was observed at every time point (Fig. 2A). The major decrease was observed after the incubation with UA and MIX, obtaining on average a reduction of $60 \%$ for both 9 and 12 hours. Androgen receptor protein levels were also determined in LNCaP cells treated with urolithins, inducing a decrease between $50 \%$ and $60 \%$ (Fig. 2B).

\subsection{Urolithins A and B inhibit the PSA promoter activity}

To assess whether urolithins affected the transcriptional activation of PSA, transient transfections in PC3 cells using a luciferase reporter vector carrying $6 \mathrm{~kb}$ of the PSA promoter were performed. ${ }^{47}$ PC3 cells were chosen because they are PSA negative and although they are considered AR-negative they do express low AR mRNA and protein levels ${ }^{48}$ in addition to retaining co-regulators necessary for AR activity in prostate tumor progression. ${ }^{49}$ Therefore, changes in PSA promoter activity would be accurately reflected after incubation with urolithins and/or DHT in these reporter assays. Six hours after transfection with the reporter vector, cells were incubated with urolithins, either in the absence or in the presence of DHT. As expected, treatment with $1 \mathrm{nM}$ DHT increased the luciferase activity by $83 \%$ compared to cells incubated in the absence of DHT, which exhibited similar activity to the basic pGL3 vector (Fig. 3). DHT-incubated cells treated with UA, UB or MIX showed a reduction in luciferase activity. UA-incubated cells showed a slightly higher inhibition in luciferase activity than UB and MIX when compared to the DHT-induced promoter, although this was not statistically
A)



B)


Fig. 1 (A) PSA mRNA levels determined by real time RT-PCR. Bars represent PSA mRNA levels in LNCaP cells either control (0.10\% of DMSO) or incubated with UA, UB or MIX. The different incubation conditions are indicated in the figure. Results are expressed in fold changes compared to the untreated cells and normalized using APRT as an endogenous control. They are the mean \pm SE of 3 different experiments. $* \star * p<0.001$. (B) Determination of PSA protein levels by Western blotting. Bars represent PSA protein levels in LNCaP cells either control $(0.10 \%$ of DMSO) or incubated with UA, UB or MIX. Results are expressed in fold changes compared to the untreated cells and represent the mean \pm SE of 3 different experiments. ${ }^{* \star} p$ $<0.001$. 
A)

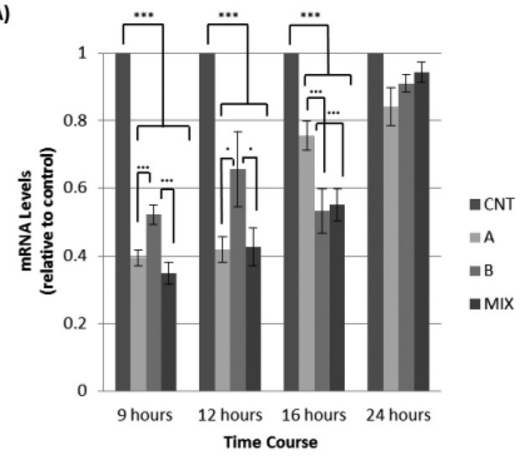

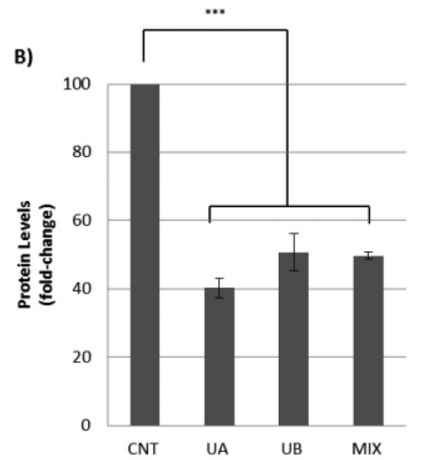

\begin{tabular}{|c|c|}
\hline $\begin{array}{c}\text { AR } \\
\text { (110 kDa) }\end{array}$ & - \\
\hline $\begin{array}{c}\text { Tubulin } \\
\text { (50 kDa) }\end{array}$ & \\
\hline
\end{tabular}

Fig. 2 (A) AR mRNA levels determined by real time RT-PCR. Incubation conditions are the same as described in Fig. 1A. Results are expressed in fold changes compared to the untreated cells and normalized using APRT as an endogenous control. They are the mean \pm SE of 3 different experiments. ${ }^{* *} p<0.001$. (B) Determination of AR protein levels by Western blotting. Results are expressed in fold changes compared to the untreated cells and represent the mean $\pm \mathrm{SE}$ of 3 different experiments. ${ }^{* *} p<0.001$.

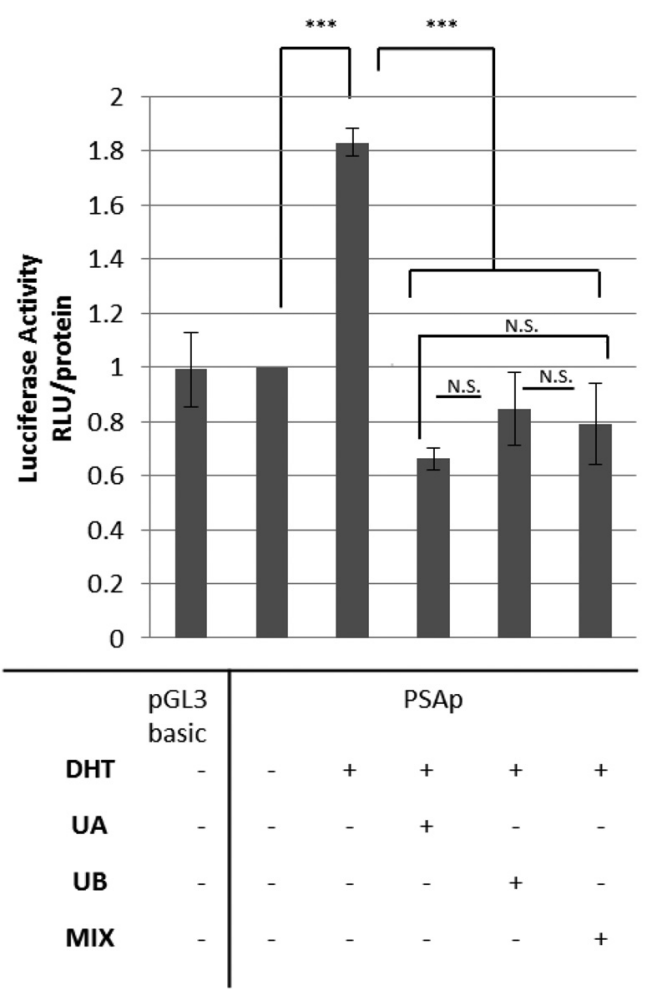

Fig. 3 PSA promoter activity in PC3 cells. Cells were transfected with a luciferase reporter vector carrying $6 \mathrm{~kb}$ of the PSA promoter, and $6 \mathrm{~h}$ later they were treated with UA, UB and MIX in the presence or absence of $1 \mathrm{nM}$ DHT. Results are expressed as luciferase relative units/total protein compared to control. They are the mean \pm SE of 3 different experiments. ${ }^{* *} p<0.001$. N.S., not significant.

significant (Fig. 3). These results indicated a repression of DHTinduced PSA promoter activation by urolithins. Cells incubated only with UA, UB or MIX exhibited basal luciferase activity, similar to the activity observed for pGL3 and PSAp in the absence of DHT (inactive PSAp, data not shown).

\subsection{PSA expression correlates with the binding of nuclear extracts to an ARE}

The regulation of PSA by androgens takes place through the ARE sequences in its promoter region. ${ }^{38}$ The effect of
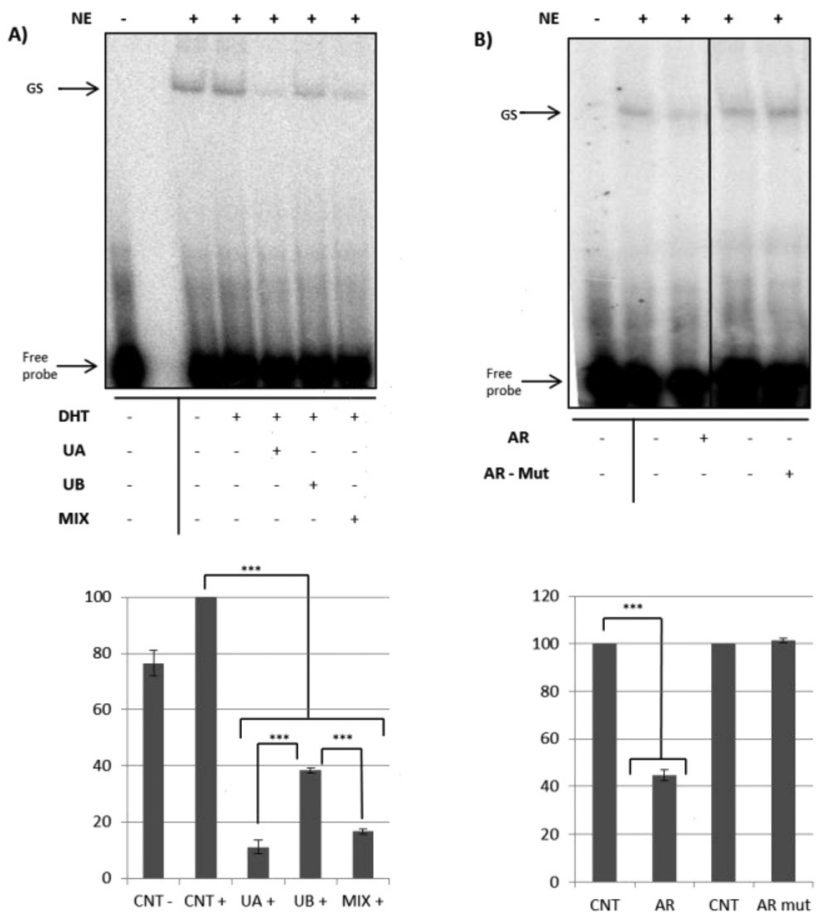

Fig. 4 (A) Effect of urolithins on AR binding to nuclear proteins. EMSA was performed using the $A R$ consensus sequence as a probe and nuclear extracts from LNCaP cells. First lane corresponds to the probe alone. Nuclear extracts were either control or treated cells with $1 \mathrm{nM}$ DHT and $40 \mu \mathrm{M}$ of UA, UB or MIX for 24 hours. ${ }^{* *} p<0.001$. N.S., not significant. (B) Competition assays. The binding of untreated LNCaP nuclear extracts to the AR consensus sequence was competed with the addition of either $3 \mathrm{ng}$ ( 5 -fold excess) of unlabeled AR or unlabeled mutated AR in the binding reaction. 
A)

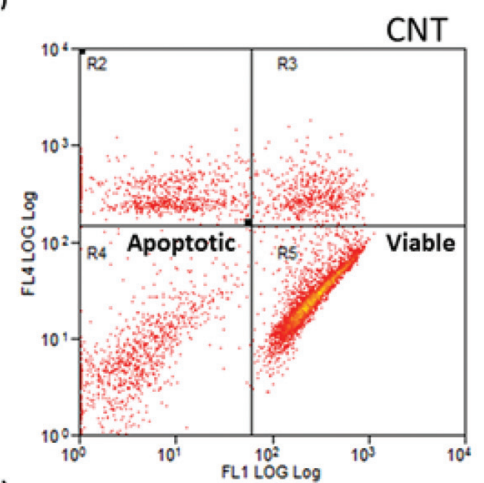

B)

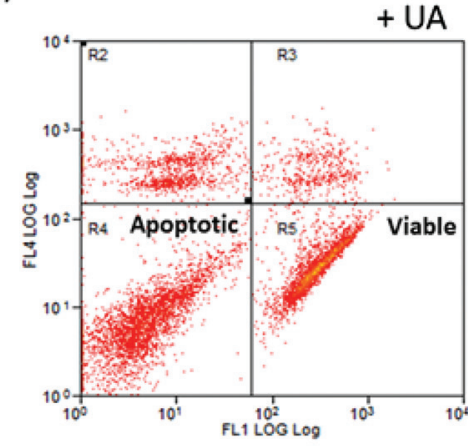

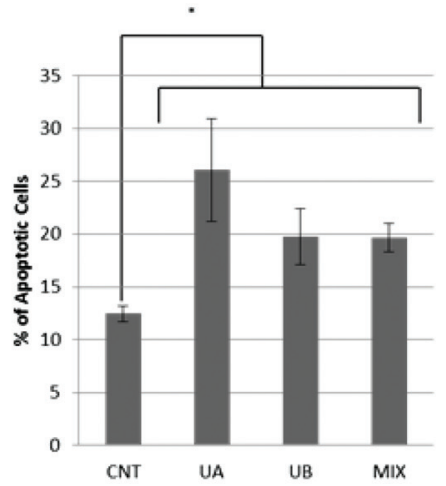

D)

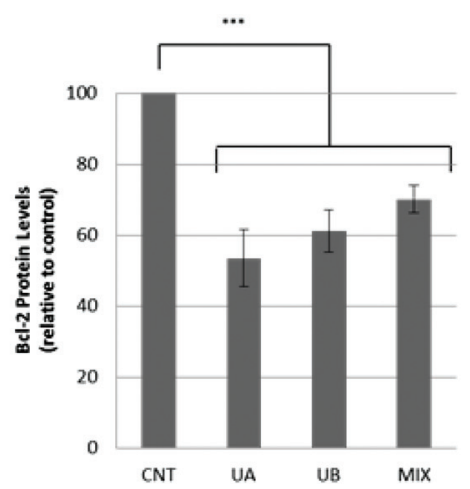

Fig. 5 Apoptosis determined by flow cytometry. (A) Representative flow cytometer histograms displaying the cell population in the untreated control sample (0.12\% DMSO) and (B) in cells incubated with $40 \mu \mathrm{M}$ UA. (C) Percentage of apoptotic cells determined by flow cytometry. Bars represent $\mathrm{LNCaP}$ cells either untreated control $(0.12 \%$ of DMSO), or incubated with UA, UB or MIX ( $40 \mu M)$ after 24 hour of exposure. Results represent the mean \pm SE of 3 different experiments. ${ }^{*} p<0.05$. (D) Bcl-2 protein levels in cells incubated with UA, UB or MIX for 24 hours. ${ }^{* * *} p<0.001$.

urolithins on ARE binding to nuclear proteins was determined using electrophoretic mobility shift assays (EMSAs). EMSAs were performed using an ARE consensus sequence as the probe and nuclear extracts from untreated (control) and treated LNCaP cells. Cells were incubated with $1 \mathrm{nM}$ DHT and urolithins for 24 hours. As shown in Fig. 4A, the interaction of nuclear extracts from control cells with the radiolabelled probe originated a shifted band, the intensity of which was clearly decreased upon incubation with UA, UB or MIX. The highest reduction was seen after UA and MIX incubation (Fig. 4A), with an $85 \%$ decrease in band intensity, followed by UB.

To assess the binding specificity, $3 \mathrm{ng}$ of unlabeled consensus ARE (corresponding to a 5 -fold excess compared to the probe) or unlabeled mutated AR were added to the binding reaction. As shown in Fig. 4B, a 56\% reduction in band intensity was observed when the binding to the ARE labeled probe was competed with the unlabeled probe, whereas the competition with the unlabeled mutated AR did not affect the intensity of the shifted band.

\subsection{Urolithins A and B induce apoptosis and a decrease of Bcl-2 protein levels in LNCaP cells}

We examined the effect of urolithins on apoptosis using the rhodamine method. Following incubation of LNCaP cells with UA, UB and MIX for 24 hours, the percentage of apoptotic cells increased compared with the control (0.20\% DMSO). A 14\% increase in apoptotic cell population was observed after incubation with UA, whereas UB and MIX caused a 7\% increase, although this difference was not statistically significant (Fig. 5A-5C). Both in vitro and in vivo studies have established that $\mathrm{Bcl}-2$ expression confers anti-apoptotic activity in prostate cancer and its overexpression is linked to progression into advanced prostate cancer. ${ }^{50} \mathrm{Bcl}-2$ overexpression has also been correlated with high PSA levels in prostate cancer. ${ }^{36}$ Hence, Bcl-2 protein levels were measured after urolithin incubation. UA, UB and MIX induced a decrease in Bcl-2 protein levels after 24 hour treatment (Fig. 5D).

\section{Discussion}

The main objective of our study was to determine the potential role of the major polyphenol metabolites in walnuts, urolithins, in the modulation of the prostate-specific antigen and the androgen receptor in prostate cancer cells. A great number of dietary components such as lycopene, vitamin E, selenium, isoflavones and polyphenols potentially affect a range of carcinogenic pathways in the prostate, including androgen metabolism, cell cycle processes and apoptosis, maintenance of mitochondrial membrane potentials, insulin-like growth factor 
(IGF)-Akt signaling, and response to oxidative stress. $^{51}$ Although, these dietary components have been assessed for their chemo-preventive capacities, there are a limited number of studies focusing on the role that walnut polyphenols have in the prevention of prostate cancer, especially those addressed to elucidate the molecular mechanisms involved. As an example, the anticancer activity for urolithins A, B, C, and D through the inhibition of CYP1B1 $1^{52}$ was reported in human prostate carcinoma $22 \mathrm{Rv} 1$ cells, while pedunculagin, ${ }^{30}$ ellagic acid $^{42}$ and extracts from the green husk of walnuts ${ }^{53}$ showed an anti-proliferative and apoptotic effect on LNCaP cells. Other authors have also observed that urolithins localized to the mouse prostate gland and inhibited the growth of both androgen-dependent and androgen-independent prostate cancer cell lines. $^{54}$

In our study, there was a clear repression of PSA transcription by urolithins A and B, as well as a decrease in PSA protein levels. The aforementioned decrease in PSA levels upon treatment with urolithins correlated with the down-regulation of the androgen receptor. The decrease in PSA and AR levels after incubation with urolithins is in agreement with the effects reported for other phenolic compounds, such as epigallocatechin gallate (EGCG), grape seed procyanidins or caffeic acid. PSA and AR play a pivotal role in prostate cancer development and progression and a potential cross-talk between these two genes has been postulated by several authors. ${ }^{55,56}$ In a recent study, Saxena et al. (2012) ${ }^{55}$ pointed out that PSA is not only a biomarker of prostate cancer and a known downstream target of the androgen receptor, but it is also required for AR mRNA and protein expression. Similarly, several authors reported that $\mathrm{AR}$ inhibition resulted in a marked decrease in cell proliferation. ${ }^{57,58}$ Thus, the observed effect of urolithins on prostate cancer cells could be due to their possible interference in this cross-talk and the reduction of PSA and AR levels.

A possible manner by which urolithins could interfere with the previously mentioned AR-PSA cross-talk is by displaying phytoestrogen-like activity. In this direction, the modulation of hormone receptors by phenolic dietary components, such as isoflavones, has been widely studied. Some authors compared urolithins with several phytoestrogens, such as genistein, daidzein, resveratrol and enterolactone. These authors reported an interference caused by urolithins in endocrine pathways proposing them as possible phytoestrogens. ${ }^{29}$ In this sense, the estrogenic and anti-estrogenic activity reported for urolithins has been related to their interaction with the estrogen receptor in human breast cancer cells (MCF-7). ${ }^{29}$ However, phytoestrogens, such as genistein, do not only interact with the estrogen receptor but have also been shown to decrease $\mathrm{AR}$ levels in LNCaP cells, and to cause a reduction in the binding of nuclear proteins to an ARE. ${ }^{59}$ Considering all of the above, we explored if urolithins were able to exert an effect over the androgen receptor, similar to that induced by phytoestrogens. In our study we demonstrate that the activation of the PSA promoter by DHT was blocked upon incubation with urolithins. The reduced activation of PSA, which is an AR-regulated promoter, could be explained by the decrease in the binding of nuclear proteins to a consensus ARE, which under our conditions was an $86 \%$ reduction. In addition, the binding of AR to AREs was reduced by $32 \%$ after direct incubation of untreated nuclear proteins with UA (data not shown). Hence, the decrease in transcription caused by urolithins over PSA levels was due to a direct effect on the PSA promoter. Our results are in keeping with those observed by Larrosa $(2006)^{28,29}$ and colleagues who studied the binding affinity of urolithins to the estrogen receptor in breast adenocarcinoma cells. These authors observed a higher binding affinity for urolithin A than for urolithin B, similar to our results in which a higher decrease in binding was observed upon incubation with UA when compared to UB.

It is important to note that PSA is fundamental in the pathophysiology of prostate cancer. It stimulates oxidative stress in LNCaP and PC3 cells, ${ }^{60}$ and is also involved in tumor invasion and metastasis. ${ }^{61}$ Considering its role in prostate cancer progression, we explored if the pronounced decrease of PSA levels upon urolithin incubation would result in an increase in apoptosis. Urolithins indeed caused an increase in apoptotic activity in LNCaP cells. Moreover, the increase in apoptosis upon incubation with urolithins was correlated with a decrease in the expression of Bcl-2, a critical regulator of the apoptotic pathway, and a potent suppressor of apoptosis. ${ }^{62}$ Human tumors usually express high levels of Bcl-2 protein and in prostate cancer its levels correlate with high levels of PSA. ${ }^{36}$ Thus, the decrease in Bcl-2 and the apoptotic activity induced upon incubation with urolithins could be linked to the inhibition of PSA by these compounds. Other authors have reported that juglone, a non-polyphenolic compound found in roots and leaves of the walnut tree, can cause apoptosis in prostate cancer cells, in this case, associated with mitochondrial dysfunction and activation of caspases 3 and $9 .^{63}$

\section{Conclusion}

Our results provide new insights into the effect metabolites of a common dietary component have on molecular mechanisms involved in prostate carcinogenesis, which could in turn provide a foundation for developing strategies for disease prevention. The effect of dietary agents on cancer can be used to identify molecular therapeutic targets, and used as part of a chemo-preventive strategy. Dietary intervention targeting multiple pathways might, therefore, be an effective therapeutic approach, either alone, or in conjunction with targeted pharmaceutical agents.

In summary, we demonstrated a reduction in PSA and AR levels induced by urolithins. This effect could be due to a decreased binding of the AR to AREs, and to decreased levels of the androgen receptor resulting in PSA transcription inhibition. An induction of apoptosis in LNCaP cells was also observed, which may be caused by the down-regulation of AR and PSA, as well as a decrease of Bcl-2 protein levels. A diet high in ET-rich foods, such as walnuts, provides a considerable 
intake of pedunculagin and its metabolites, urolithins, which could assist in the prevention of prostate cancer in men.

\section{Conflict of interest}

The authors have no potential conflict of interest.

\section{Acknowledgements}

The $6 \mathrm{~kb}$ PSA promoter construct containing three AREs in front of a luciferase reporter gene (PSAp) was graciously provided by Dr Charles Young from the Mayo Clinic, Rochester, MN.

This work was supported by grants from the California Walnut Commission (FBG-306913) and the Spanish Government of Science and Innovation (SAF2011-23582). C.S.G. was supported by scholarships from the "Consejo Nacional de Ciencia y Tecnología, CONACYT" and the "Dirección General de Relaciones Internacionales de la Secretaría de Educación Pública, DGRI-SEP" from Mexico. Our group holds the Quality Mention from the Government of Catalonia, Spain (SGR2009118).

\section{References}

1 American Cancer Society, Am. Cancer Soc., 2011, 9-18.

2 M. Stacewicz-Sapuntzakis, G. Borthakur, J. L. Burns and P. E. Bowen, Mol. Nutr. Food Res., 2008, 52, 114-130.

3 J. Dai and R. J. Mumper, Molecules, 2010, 15, 7313-7352.

4 S. Ramos, Mol. Nutr. Food Res., 2008, 52, 507-526.

5 P. Signorelli and R. Ghidoni, J. Nutr. Biochem., 2005, 16, 449-466.

6 R. Tsao, Nutrients, 2010, 2, 1231-1246.

7 D. T. Verhoeven, H. Verhagen, R. A. Goldbohm, P. A. van den Brandt and G. van Poppel, Chem.-Biol. Interact., 1997, 103, 79-129.

8 S. J. Duthie and V. L. Dobson, Eur. J. Nutr., 1999, 38, 28-34.

9 R. W. Owen, A. Giacosa, W. E. Hull, R. Haubner, B. Spiegelhalder and H. Bartsch, Eur. J. Cancer, 2000, 36, 1235-1247.

10 N. Khan, F. Afaq, M. Saleem, N. Ahmad and H. Mukhtar, Cancer Res., 2006, 66, 2500-2505.

11 G. Corona, M. Deiana, A. Incani, D. Vauzour, M. A. Dessì and J. P. E. Spencer, Biochem. Biophys. Res. Commun., 2007, 362, 606-611.

12 G. Corona, M. Deiana, A. Incani, D. Vauzour, M. A. Dessì and J. P. E. Spencer, Mol. Nutr. Food Res., 2009, 53, 897903.

13 W. Wang, L. Heideman, C. S. Chung, J. C. Pelling, K. J. Koehler and D. F. Birt, Mol. Carcinog., 2000, 28, 102110.

14 S. K. Mantena, M. S. Baliga and S. K. Katiyar, Carcinogenesis, 2006, 27, 1682-1691.
15 R. Fabiani, A. De Bartolomeo, P. Rosignoli, M. Servili, G. F. Montedoro and G. Morozzi, Eur. J. Cancer Prev., 2002, 11, 351-358.

16 D. Vauzour, A. Rodriguez-Mateos, G. Corona, M. J. OrunaConcha and J. P. E. Spencer, Nutrients, 2010, 2, 11061131.

17 R. Thomas, M. Williams, H. Sharma, A. Chaudry and P. Bellamy, Prostate Cancer Prostatic Dis., 2014, 17, 180186.

18 L. Fini, E. Hotchkiss, V. Fogliano, G. Graziani, M. Romano, E. B. De Vol, H. Qin, M. Selgrad, C. R. Boland and L. Ricciardiello, Carcinogenesis, 2008, 29, 139-146.

19 S. U. Mertens-Talcott, P. Jilma-Stohlawetz, J. Rios, L. Hingorani and H. Derendorf, J. Agric. Food Chem., 2006, 54, 8956-8961.

20 V. Granci, Y. M. Dupertuis and C. Pichard, Curr. Opin. Clin. Nutr. Metab. Care, 2010, 13, 417-422.

21 A. K. Garg, T. A. Buchholz and B. B. Aggarwal, Antioxid. Redox Signaling, 2005, 7, 1630-1647.

22 R. Estruch, E. Ros, J. Salas-Salvadó, M.-I. Covas, D. Corella, F. Arós, E. Gómez-Gracia, V. Ruiz-Gutiérrez, M. Fiol, J. Lapetra, R. M. Lamuela-Raventos, L. Serra-Majem, X. Pintó, J. Basora, M. A. Muñoz, J. V. Sorlí, J. A. Martínez and M. A. Martínez-González, N. Engl. J. Med., 2013, 368, 1279-1290.

23 A. Pan, Q. Sun and J. Manson, J. Nutr., 2013, 512-518.

24 E. Ros, I. Núñez, A. Pérez-Heras, M. Serra, R. Gilabert, E. Casals and R. Deulofeu, Circulation, 2004, 109, 16091614.

25 J. A. Vinson and Y. Cai, Food Funct., 2012, 3, 134-140.

26 J. Regueiro, C. Sánchez-González, A. Vallverdú-Queralt, J. Simal-Gándara, R. Lamuela-Raventós and M. IzquierdoPulido, Food Chem., 2014, 152, 340-348.

27 J. M. Landete, Food Res. Int., 2011, 44, 1150-1160.

28 M. Larrosa, F. A. Tomás-Barberán and J. C. Espín, J. Nutr. Biochem., 2006, 17, 611-625.

29 M. Larrosa, A. González-Sarrías, M. T. García-Conesa, F. A. Tomás-Barberán and J. C. Espín, J. Agric. Food Chem., 2006, 54, 1611-1620.

30 A. González-Sarrías, J. A. Giménez-Bastida, M. T. GarcíaConesa, M. B. Gómez-Sánchez, N. V. García-Talavera, A. GilIzquierdo, C. Sánchez-Alvarez, L. O. Fontana-Compiano, J. P. Morga-Egea, F. A. Pastor-Quirante, F. Martínez-Díaz, F. A. Tomás-Barberán and J. C. Espín, Mol. Nutr. Food Res., 2010, 54, 311-322.

31 J. C. Espín, M. Larrosa, M. T. García-Conesa and F. TomásBarberán, Evid. Based Complement. Alternat. Med., 2013, 2013, 270418.

32 M. Carvalho, P. J. Ferreira, V. S. Mendes, R. Silva, J. A. Pereira, C. Jerónimo and B. M. Silva, Food Chem. Toxicol., 2010, 48, 441-447.

33 W. E. Hardman, G. Ion, J. A. Akinsete and T. R. Witte, Nutr. Cancer, 2011, 63, 960-970.

34 M. Stolarczyk, J. P. Piwowarski, S. Granica, J. Stefańska, M. Naruszewicz and A. K. Kiss, Phytother. Res., 2013, 27, 1842-1848. 
35 K. B. Cleutjens, H. A. van der Korput, C. C. van Eekelen, H. C. van Rooij, P. W. Faber and J. Trapman, Mol. Endocrinol., 1997, 11, 148-161.

36 S. Altuwaijri, J. Cancer Ther., 2012, 03, 331-336.

37 F. Yeung, X. Li, J. Ellett, J. Trapman, C. Kao and L. W. Chung, J. Biol. Chem., 2000, 275, 40846-40855.

38 S. P. Balk, J. Clin. Oncol., 2003, 21, 383-391.

39 A. Magklara, A. Scorilas and C. Stephan, Urology, 2000, 2, 527-532.

40 Z. Sun, J. Pan and S. P. Balk, Nucleic Acids Res., 1997, 25, 3318-3325.

41 P. Oettgen, E. Finger and Z. Sun, J. Biol. Chem., 2000, 275, 1216-1225.

42 R. Vicinanza, Y. Zhang, S. M. Henning and D. Heber, Evid. Based Complement Alternat. Med., 2013, 2013, 247504.

43 A. González-Sarrías, J.-C. Espín, F. A. Tomás-Barberán and M.-T. García-Conesa, Mol. Nutr. Food Res., 2009, 53, 686-698.

44 C. Oleaga, C. J. Ciudad, M. Izquierdo-Pulido and V. Noé, Mol. Nutr. Food Res., 2013, 57, 986-995.

45 N. C. Andrews and D. V. Faller, Nucleic Acids Res., 1991, 19, 2499.

46 L. Rodríguez, X. Villalobos, S. Dakhel, L. Padilla, R. Hervas, J. L. Hernández, C. J. Ciudad and V. Noé, Biochem. Pharmacol., 2013, 86, 1541-1554.

47 N. Xing, Y. Chen, S. Mitchell and C. Young, Carcinogenesis, 2001, 22, 409-414.

48 F. Alimirah, J. Chen, Z. Basrawala, H. Xin and D. Choubey, FEBS Lett., 2006, 580, 2294-2300.

49 I. V. Litvinov, L. Antony, S. L. Dalrymple, R. Becker, L. Cheng and J. T. Isaacs, Prostate, 2006, 1338.

50 K. Chaudhary, P. Abel and E. Lalanil, Environ. Health Perspect., 1999, 107, 49-57.
51 V. Venkateswaran and L. H. Klotz, Nat. Rev. Urol., 2010, 7, 442-453.

52 S. G. Kasimsetty, D. Bialonska, M. K. Reddy, C. Thornton, K. L. Willett and D. Ferreira, J. Agric. Food Chem., 2009, 57, 10636-10644.

53 A. A. Alshatwi, T. N. Hasan, G. Shafi, N. A. Syed, A. H. AlAssaf, M. S. Alamri and A. S. Al-Khalifa, Evid. Based Complement Alternat. Med., 2012, 2012, 103026.

54 N. P. Seeram, W. J. Aronson, Y. Zhang, S. M. Henning, A. Moro, R.-P. Lee, M. Sartippour, D. M. Harris, M. Rettig, M. A. Suchard, A. J. Pantuck, A. Belldegrun and D. Heber, J. Agric. Food Chem., 2007, 55, 7732-7737.

55 P. Saxena, M. Trerotola, T. Wang, J. Li, A. Sayeed, J. Vanoudenhove, D. S. Adams, T. J. Fitzgerald, D. C. Altieri and L. R. Languino, Prostate, 2012, 72, 769-776.

56 Y. Niu, S. Yeh, H. Miyamoto, G. Li, S. Altuwaijri, J. Yuan, R. Han, T. Ma, H. Kuo and C. Chang, Cancer Res., 2008, 68, 7110-7119.

57 L. Kong, Q. Yuan, H. Zhu, Y. Li, Q. Guo, Q. Wang, X. Bi and X. Gao, Biomaterials, 2011, 32, 6515-6522.

58 H. L. Oh and C.-H. Lee, Bioorg. Med. Chem. Lett., 2011, 21, 1347-1349.

59 J. N. Davis, O. Kucuk and F. H. Sarkar, Mol. Carcinog., 2002, 34, 91-101.

60 S. Williams, P. Singh, J. Isaacs and S. Denmeade, Prostate, 2007, 329, 312-329.

61 M. M. Webber, A. Waghray and D. Bello, Clin. Cancer Res., 1995, 1, 1089-1094.

62 A. Malik, S. Afaq, M. Shahid, K. Akhtar and A. Assiri, Asian Pac. J. Trop. Med., 2011, 4, 550-555.

63 H. Xu, X. Yu, S. Qu and D. Sui, Bioorg. Med. Chem. Lett., 2013, 23, 3631-3634. 